\title{
Ursodeoxycholic acid-induced inhibition of DLC1 protein degradation leads to suppression of hepatocellular carcinoma cell growth
}

\author{
GOH EUN CHUNG ${ }^{1}$, JUNG-HWAN YOON ${ }^{2}$, JEONG-HOON LEE ${ }^{2}$, HWI YOUNG KIM ${ }^{2}$, \\ SUN JUNG MYUNG ${ }^{2}$, SU JONG YU ${ }^{2}$, SUNG-HEE LEE ${ }^{2}$, SOO-MI LEE ${ }^{2}$, YOON JUN KIM ${ }^{2}$ and HYO-SUK LEE ${ }^{2}$ \\ ${ }^{1}$ Department of Internal Medicine, Gangnam Healthcare Center, Seoul National University Hospital; ${ }^{2}$ Department of \\ Internal Medicine and Liver Research Institute, Seoul National University College of Medicine, Seoul, Republic of Korea
}

Received January 14, 2011; Accepted March 4, 2011

DOI: 10.3892/or.2011.1239

\begin{abstract}
Ursodeoxycholic acid (UDCA), a hydrophilic bile acid, has been shown to inhibit mitogenic signaling and suppressing cell proliferation in colonic tumorigenesis. The transcription of $D L C l$ (deleted in liver cancer), a tumor suppressor gene, is frequently silenced in various types of human cancer. In this study, we postulated that UDCA may inhibit DLC1 protein degradation in hepatocellular carcinoma (HCC) cells, and increased DLC1 expression may suppress HCC cell growth. Human HCC cell lines were used in this study. The methylation status was measured by methylation-specific PCR following sodium bisulfite treatment. Cell proliferation was assessed using an MTS assay. Kinase signaling cascades were evaluated by immunoblot analysis. For assessing ubiquitination, immunoprecipitation analysis was used. To inhibit cellular protein, specific small interfering RNAs (siRNAs) were transfected into cells. DLC1 protein levels increased over time following UDCA treatment. Specifically, UDCA increased the half-life of the DLC1 protein by inhibiting proteasomal degradation of DLC1 without affecting ubiquitination of the DLC1 protein. In addition, HCC cell growth was suppressed following UDCA
\end{abstract}

Correspondence to: Professor Jung-Hwan Yoon, Department of Internal Medicine, Seoul National University College of Medicine, 28 Yungun-dong, Chongno-gu, Seoul 110-744, Republic of Korea E-mail: yoonjh@snu.ac.kr

Abbreviations: MAPK, mitogen-activated protein kinase; UDCA, ursodeoxycholic acid; HCC, hepatocellular carcinoma; GAP, GTPaseactivating protein; DMEM, Dulbecco's modified Eagle's medium; MTS, 3,4-(5-dimethylthiazol-2-yl)-5-(3-carboxymethoxyphenyl)-2(4-sulfophenyl)-2H-tetrazolium salt; JNK, c-Jun N-terminal kinase; MSP, methylation-specific PCR; siRNA, short interfering RNA; Ub, ubiquitination

Key words: hepatocellular carcinoma, ursodeoxycholic acid, deleted in liver cancer 1 treatment and this growth suppression was significantly reversed following transfection with DLC1-siRNA. Inhibition of DLC1 increased cellular proliferation; this was reduced after Rho-inhibitor treatment. Finally, RhoA activity was reduced following UDCA treatment; this result was reversed and thus increased following DLC1-siRNA transfection. In conclusion, these results demonstrate that UDCA induces DLC1 protein expression by inhibiting proteasomal DLC1 degradation in a ubiquitin-independent manner, and that DLC1 induction participates in UDCA-induced suppression of HCC cell growth. These observations implicate UDCA as an anti-proliferative agent in HCC.

\section{Introduction}

Bile acids can activate a variety of signals depending on specific cell types (1). In particular, bile acids have been implicated in biliary carcinogenesis by altering intracellular signaling and regulatory genes. For example, bile acids may block Mcl-1 protein degradation via an epidermal growth factor receptor/Raf-1-dependent mechanism in cholangiocarcinoma cells (2). In addition, bile acids can induce cyclooxygenase- 2 expression via the mitogen-activated protein kinase (MAPK) cascade in cholangiocarcinoma cells (3). However, in contrast to biliary tract epithelial cells, which are physiologically exposed to extremely high concentrations of bile acids, hepatocytes undergo cell death upon exposure to bile acids, particularly hydrophobic bile acids such as deoxycholic acid (4). Unlike these bile acids, however, ursodeoxycholic acid (UDCA) is a relatively hydrophilic bile acid and is well known to have a hepatocellular protective effect in a variety of liver diseases (5).

In colonic tumorigenesis, UDCA has been shown to inhibit mitogenic signaling and thereby suppress cell growth (6), suggesting that this bile acid might be used as an antiproliferative agent. However, the mechanism by which UDCA functions is still unclear, and its effect has not yet been tested in hepatocellular carcinoma (HCC). Since UDCA is not toxic to hepatocytes and may even have a hepatoprotective effect, it would be more desirable if this bile acid could suppress HCC cellular growth without affecting the viability of hepatocytes. 
The DLC1 (deleted in liver cancer) gene at 8p22 encodes a Rho GTPase-activating protein (GAP) and is expressed in most human tissues (7). DLC1 can activate GTP-bound and inactivate GDP-bound proteins to play a role in the function of Rho protein (8). It has been reported that the expression of DLC1 is frequently down-regulated or silenced in various types of human cancer $(9,10)$. Transcriptional silencing of DLC1 by aberrant promoter methylation is commonly involved in the pathogenesis of breast, lung, colon, prostate, gastric and liver cancers $(11,12)$. Moreover, several studies have now shown the tumor suppressive roles of DLC1, which suppresses cellular proliferation and invasion in $\operatorname{HCC}(13,14)$. While the protein expression of DLC1 is transcriptionally suppressed, it is anticipated that its expression can be increased if protein degradation is inhibited by a certain mechanism. Since our previous study demonstrated that bile acids can block protein degradation (2), we postulated the following: i) UDCA may inhibit DLC1 protein degradation in HCC cells, thereby increassing its expression and ii) increased DLC1 expression may suppress HCC cellular growth. Collectively, the results of the current study demonstrate that UDCA induces DLC1 protein expression by inhibiting proteasomal DLC1 degradation in an ubiquitin-independent manner, and that DLC1 induction participates in UDCA-induced suppression of HCC cell growth. These observations implicate UDCA as an anti-proliferative agent in HCCs.

\section{Materials and methods}

Cell Cultures and UDCA treatment. Three human HCC cell lines were used in this study: Huh-BAT, a well differentiated HCC cell line, and SNU475 and SNU761, which are poorly differentiated HCC cell lines. Cells were grown in Dulbecco's modified Eagle's medium (DMEM) supplemented with $10 \%$ fetal bovine serum, 100,000 U/liter penicillin and $100 \mathrm{mg} / \mathrm{l}$ streptomycin at $37^{\circ} \mathrm{C}$ in a humidified atmosphere of $5 \% \mathrm{CO}_{2}$ and $95 \%$ air. The immortalized human hepatocytes (15) were maintained over 150 passages in a chemically defined Ham's F-12 medium (Sigma Chemical Co., St. Louis, MO) containing $50 \mathrm{ng} / \mathrm{ml}$ epidermal growth factor (Collaborative Biomedical, Waltham, MA), $10 \mu \mathrm{g} / \mathrm{ml}$ insulin, $5 \mu \mathrm{g} / \mathrm{ml}$ transferring, $3.5 \mu \mathrm{g} / \mathrm{M}$ hydrocortisone, $2 \mathrm{mM}$ glutamine, $0.1 \mu \mathrm{M}$ $\mathrm{CuSO}_{4} \cdot 5 \mathrm{H}_{2} \mathrm{O}, 0.03 \mu \mathrm{M} \mathrm{H}_{2} \mathrm{SeO}_{3}, 0.5 \mathrm{nM} \mathrm{ZnSO}_{4} \bullet 7 \mathrm{H}_{2} \mathrm{O}$, $1 \mathrm{ng} / \mathrm{ml}$ glucagon, $6.5 \mathrm{ng} / \mathrm{ml}$ growth hormone and $0.5 \mu \mathrm{g} /$ ml linoleic acid (Sigma Chemical Co.). We used UDCA, which is a secondary hydrophilic bile acid, and chenodeoxycholate (CDC), a hydrophobic bile acid, for this study (Sigma Chemicals Co.).

Reverse transcription PCR. Total RNA was extracted from cells using TRIzol Reagent (Invitrogen, Carlsbad, CA). The resulting cDNA template was prepared using oligo random primers and Moloney Murine Leukemia Virus reverse transcripts as described previously (16). After the reverse transcription reaction, the cDNA template was amplified by PCR with Taq polymerase (Invitrogen). Expression of the DLC1 cDNA was assessed by using PCR technology and the following primers: 5'-CTC GCG AAC AAG GGA TTA TG-3' (forward) and 5'-ACA CAG CCC AGC CAC ATT A-3' (reverse) which amplified a 507-bp fragment (17). The amplified products were separated on $1 \%$ agarose gels, stained with ethidium bromide, and photographed using ultraviolet illumination.

Sodium bisulfite treatment. DNA was denatured with $0.3 \mathrm{M}$ $\mathrm{NaOH}$ and treated with $3.6 \mathrm{M}$ sodium bisulfite solution. The bisulfite solution was prepared to be either $2.4 \mathrm{~mol} / 1$ sodium metabisulfite ( $\mathrm{pH}$ 5.0-5.2) (Sigma, St. Louis, MO)/0.5 mmol/1 hydroquinone (Sigma) for a 4-h treatment. DNA was purified using Wizard DNA Clean-Up system (Promega, Madison, WI). Purified DNA was treated with $0.3 \mathrm{M} \mathrm{NaOH}$ at $37^{\circ} \mathrm{C}$ for $15 \mathrm{~min}$, neutralized with ammonium acetate, precipitated with ethanol, and dissolved in $30 \mu 1$ Tris-EDTA buffer ( $\mathrm{pH} 8.0$ ).

Methylation-specific PCR. Bisulfite-treated DNA was amplified by methylation-specific PCR (MSP), which was able to discriminate between methylated and unmethylated alleles of the DLC1 gene. The primer sequences for the methylation specific PCR were: 5'-TTT AAA GAT CGA AAC GAG GGA GCG-3' (forward) and 5'-CCC AAC GAA AAA ACC CGA CTA ACG-3' (reverse). The sequences for unmethylation specific primers were: 5'-TTT TTT AAA GAT TGA AAT GAG GGA GTG-3' (forward) and 5'-AAA CCC AAC AAA AAA ACC CAA CTA ACA-3' (reverse) (18). The reaction was carried out under the following conditions: $95^{\circ} \mathrm{C}$ for $10 \mathrm{~min}$, followed by 40 cycles at $94^{\circ} \mathrm{C}$ for $30 \mathrm{sec}, 60^{\circ} \mathrm{C}$ for $30 \mathrm{sec}$ (for methylated reactions) or $58^{\circ} \mathrm{C}$ (for unmethylated reactions), $72^{\circ} \mathrm{C}$ for $30 \mathrm{sec}$, and a final extension at $72^{\circ} \mathrm{C}$ for $5 \mathrm{~min}$.

Cell proliferation. Cell growth was measured colorimetrically using the CellTiter $96^{\circledR}$ AQueous One Solution Cell Proliferation Assay (Promega Corporation, Madison, WI, USA), which is based on the cellular conversion of 3,4-(5-dimethylthiazol-2-yl)-5-(3-carboxymethoxyphenyl)-2(4-sulfophenyl)-2H-tetrazolium salt (MTS) into soluble form by dehydrogenase enzymes produced only in metabolically active, proliferating cells. Following each treatment, $20 \mu \mathrm{l}$ of CellTiter 96 AQueous One Solution reagent was added into each well of a 96-well plate. After 2-h incubation period, the absorbance of each well at a wavelength of $490 \mathrm{~nm}$ was recorded using an ELISA plate reader (Molecular Devices, Sunnyvale, CA, USA).

Materials and reagents. Cycloheximide was from Sigma. The MAPK inhibitors, U0126 for p42/44 MAPK and SP600125 for JNK inhibitor (Biomol Research Laboratories, Plymouth Meeting, PA), and the proteasome inhibitor, bortezomib (Janssen-Cilag International NV) were used. An adenylate cyclase inhibitor, cis-N-[2-phenylcyclopentyl]-azacyclotridec1-en-2-amine hydrochloride (MDL12330A; Sigma) was used. To silence TGR5 expression, Dharmacon siGenome (Dharmacon Inc., Lafayettes, CO, USA) was used according to the manufacturer's instructions. DLC1-short interfering RNA (siRNA) from Santa Cruz Biotechnology Inc. (Santa Cruz, CA) was used to silence DLC1 expression according to the manufacturer's instructions. Transfections were performed using siRNA Transfection Reagent (Santa Cruz Biotechnology Inc.). Rho inhibitor, Clostridium difficile toxin B-10463 (TcdB-10463), was purified as described previously (19). 
(A)

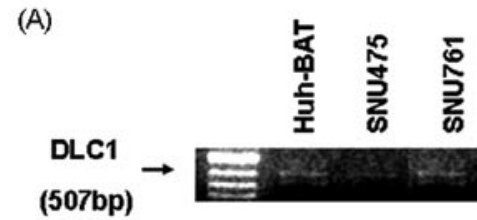

(C)

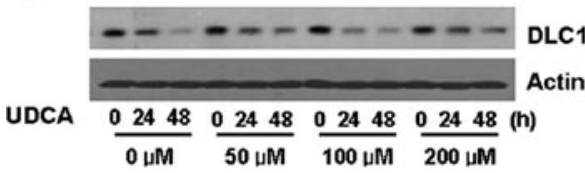

(D)

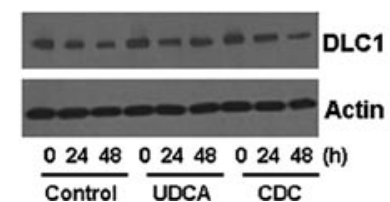

(B)

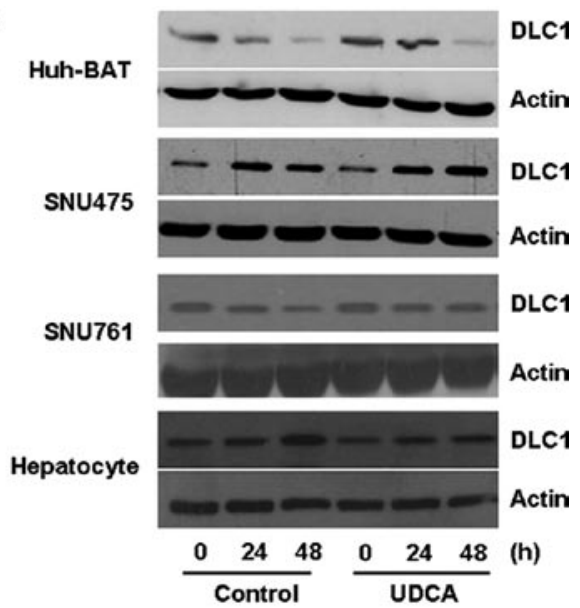

Figure 1. DLC1 transcript expression and UDCA-induced DLC1 protein expression in human HCC cell lines. (A) Total cellular RNA was isolated from Huh-BAT, SNU475 and SNU761 cells and RT-PCR analysis of DLC1 was performed. (B) HCC cells and immortalized human hepatocytes were cultured for $48 \mathrm{~h}$ in the presence and absence of UDCA $200 \mu \mathrm{M}$. Cell lysates were resolved by SDS-PAGE and immunoblot analysis was performed for DLC1. Immunoblot analysis using anti- $\beta$-actin antisera was performed as a control. (C) SNU761 cells were cultured at different concentrations of UDCA. Immunoblot analysis was performed for DLC1 and $\beta$-actin. (D) DLC1 protein expression levels were compared in SNU761 cells treated with UDCA (200 $\mu \mathrm{M}$ ) or CDC (40 $\mu \mathrm{M})$ by immunoblot analysis.

Immunoblot analysis. Cells were lysed for $20 \mathrm{~min}$ on ice with lysis buffer (50 mM Tris- $\mathrm{HCl}(\mathrm{pH} 7.4), 1 \%$ Nonidet P-40; $0.25 \%$ sodium deoxycholate, $150 \mathrm{mM} \mathrm{NaCl}, 1 \mathrm{mM}$ EDTA, $1 \mathrm{mM}$ PMSF, $1 \mu \mathrm{g} / \mathrm{ml}$ each of aprotinin, leupetin, and pepstatin; $1 \mathrm{mM} \mathrm{Na} \mathrm{VO}_{4}$, and $1 \mathrm{mM} \mathrm{NaF}$ ) and centrifuged at $14,000 \mathrm{x} \mathrm{g}$ for $10 \mathrm{~min}$ at $4^{\circ} \mathrm{C}$. Samples were separated with 10 or $12 \%$ sodium dodecyl sulfate polyacrylamide gel electrophoresis, transferred to nitrocellulose membranes, and blotted using appropriate primary antibodies with peroxidaseconjugated secondary antibodies (Biosource International, Camarillo, CA, USA). Bound antibodies were visualized using a chemiluminescent substrate (ECL; Amersham, Arlington Heights, IL, USA) and exposed to film (X-Omat; Kodak, Hannover, Germany). The primary antibodies used were: mouse monoclonal anti-DLC1 antibody purchased from BD Transduction Laboratories (Palo Alto, CA, USA), rabbit antip42/44 MAPK, rabbit anti-c-Jun N-terminal kinase (JNK), rabbit anti-phopho-p42/44 MAPK, mouse anti-phospho-JNK from Cell Signaling Technology (Beverly, MA, USA) and goat anti-actin from Santa Cruz Biotechnology Inc.

Ubiquitination of DLC1. Cells were lysed in ice-cold lysis buffer as described above, except containing $10 \mathrm{mM}$ $\mathrm{N}$-ethylmaleimide and $50 \mu \mathrm{M} \mathrm{N}$-acetyl-leucyl-leucylnorleucinal. Cell lysate were incubated with anti-DLC1 antibody overnight at $4^{\circ} \mathrm{C}$. Immune complexes were precipitated with $50 \mu \mathrm{l}$ of protein A/G PULS-Agarose (Santa Cruz Biotechnology Inc.) for $2 \mathrm{~h}$ at $4^{\circ} \mathrm{C}$ and then washed five times with lysis buffer. Polypeptides were eluted from the beads by boiling for $5 \mathrm{~min}$ in Laemmli sample buffer. The immunoprecipitates were resolved by SDS-PAGE, transferred to nitrocellulose membranes, and immunoblotted with an anti-ubiquitin antibody (Santa Cruz Biotechnology Inc.). Immune complexes on nitrocellulose were visualized and analyzed as described above. The membrane was stripped with stripping buffer (100 $\mu \mathrm{M}$ 2-mercaptoethanol; 2\% SDS; $50 \mathrm{mM}$ Tris- $\mathrm{HCl}, \mathrm{pH}$ 6.7) and reblotted with the anti-DLC1 antibody as described above.

RhoA activity assay. A glutathione-S-transferase (GST)-fusion of the Rho-GTP binding domain (RBD) of Rhotekin (amino acids 7-89), an effector of RhoA, RhoB, and RhoC was used in pull down assays to detect expression of activated RhoA-GTP as described previously (20). Total and GST-RBD-precipitated lysates were subjected to SDS-PAGE and analyzed by Western blotting using mouse anti-RhoA antibody (Cell Biolabs, Inc., CA, USA).

Data analysis. All experimental results represent at least three independent experiments using cells from a minimum of three separate isolations and are expressed as means \pm standard deviation. Comparisons between groups used a Mann-Whitney U test. All analyses used SPSS version 12.0 (SPSS Inc., Chicago, IL, USA). P $<0.05$ was considered significant.

\section{Results}

UDCA modulation of DLCl expression in human $H C C$ cells. We first used RT-PCR to examine whether DLC1 is transcriptionally expressed in different human HCC cell lines (Huh-BAT, SNU475 and SNU761). As shown in Fig. 1A, 
(A)

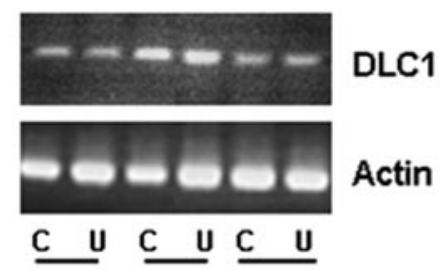

Huh-BAT SNU761 SNU475

(B)

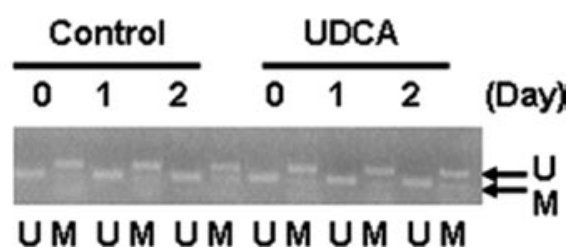

Figure 2. Methylation status of the DLC1 CpG island in HCC cell line. (A) $\mathrm{HCC}$ cells were cultured for 2 days in the presence $(\mathrm{U})$ and absence $(\mathrm{C})$ of UDCA $200 \mu \mathrm{M}$. Total cellular RNA was isolated from Huh-BAT, SNU761 and SNU475 cells and RT-PCR analysis of DLC1 was performed. (B) Bisulfite-modified DNA derived from SNU761 cell line was amplified with primers specific for unmethylated (U) or methylated (M) DNA. The 'U' and 'M' products were 178 and $172 \mathrm{bp}$, respectively.

DLC1 transcript expression was confirmed in all the cell lines examined. We next evaluated if UDCA can modulate DLC1 expression in these cell lines. DLC1 protein levels increased over time following treatment with UDCA, whereas DLC1 protein levels were not altered in immortalized human hepatocytes (Fig. 1B and C). However, hydrophobic bile acid (CDC) had no effect on DLC1 protein expression (Fig. 1D). These observations indicate that UDCA may increase DLC1 protein expression in $\mathrm{HCC}$ cells.

UDCA alteration of the methylation status of DLC1. Methylation of a $\mathrm{CpG}$ island is a genetic silencing method of a variety of tumor suppressor genes. Aberrant promoter methylation is the principal mechanism responsible for inactivation of the $D L C l$ gene in HCC cells (12). To explore the potential role of UDCA in the transcriptional silencing of the DLC1 gene, we used MSP to analyze the methylation status in HCC cells after treatment with UDCA; in MSP, methylated and unmethylated alleles are specifically amplified. As shown in Fig. 2A and B, UDCA did not alter both the mRNA levels and the methylation status of DLC1. These findings suggest that UDCA might modulate DLC1 protein levels at a posttranscriptional level.

UDCA decrease of DLC1 protein degradation. We next examined the effect of UDCA on DLC1 protein stability. In the presence of a protein translation inhibitor, cycloheximide, UDCA increased the half-life of the DLC1 protein, indicating that UDCA may suppress DLC1 protein degradation. In addition, the selective proteasome inhibitor, bortezomib, increased half-life of the DLC1 protein like in cells treated with UDCA
(Fig. 3A). The ubiquitin-proteasome pathway is a major system for the degradation of short-lived regulatory proteins (21). Since the proteasome recognizes and degrades proteins conjugated with polyubiquitin chains, we next examined whether UDCA modulates the ubiquitination of DLC1 in HCC cells. As shown in Fig. 3B, the profiles of DLC1polyubiquitin conjugates were not altered following UDCA treatment, suggesting that UDCA-induced suppression of DLC1 degradation is not mediated by the selective inhibition of polyubiquitin-DLC1 conjugates Therefore, these findings collectively indicate that UDCA induces DLC1 expression at a posttranscriptional level, specifically by inhibiting proteasomal degradation of DLC1 without affecting the methylation status of DLC1 promoter or ubiquitination of DLC1 protein.

UDCA modulation of HCC cellular proliferation. Given that UDCA decreases DLC1 protein degradation in HCC cells, we next determined whether UDCA modulates cellular proliferation of HCC cells. Indeed, HCC cellular growth was significantly suppressed following UDCA treatment in both HCC cell lines (Fig. 4A).

Involvement of TGR5 activation in UDCA-induced growth suppression in HCC cells. TGR5 is a G protein-coupled cell-surface bile acid receptor. Bile acids, which are natural agonists for TGR5, increase the intracellular cAMP levels in a dose-dependent manner (22). To determine whether UDCA-induced TGR 5 activation is an initiating signal leading to UDCA-induced growth suppression, we measured cellular proliferation in HCC cells either pre-treated with MDL12230A (adenylate cyclase inhibitor) or transfected with TGR5-specific siRNA. However, UDCA-induced cellular growth suppression was not affected by MDL12330A or TGR5-specific siRNA transfection (Fig. 4B and C). These findings suggest that cell surface TGR5 activation is not responsible for UDCA-induced growth suppression, and therefore, it is more likely that other signals activated by intracellularly transported UDCA mediate this growth suppressing effect.

DLC1 participation in UDCA-induced growth suppression. Next, we examined whether DLC1 plays a role in UDCAinduced growth suppression of HCC cells. For selective inhibition of DLC1, we transfected HCC cells with DLC1specific siRNA. As shown in Fig. 5A, UDCA-induced growth suppression was significantly reversed following DLC1-siRNA transfection as compared to the control-siRNA transfected cells. These findings indicate that UDCA-induced HCC cell growth suppression is dependent on DLC1 induction.

Kinase signals and participation in UDCA-induced growth suppression. To explore a possible mechanism of UDCAinduced DLC1-dependent growth suppression, we next examined the kinase signals regulating cell growth. DLC1 protein was effectively silenced following DLC1-specific siRNA transfection (Fig. 5B). HCC cells were treated with UDCA after either control or DLC1-siRNA transfection, and the phosphorylated status of p42/44 MAPK and JNK were assessed by immunoblot analysis. As a result, UDCA-induced 
(A)
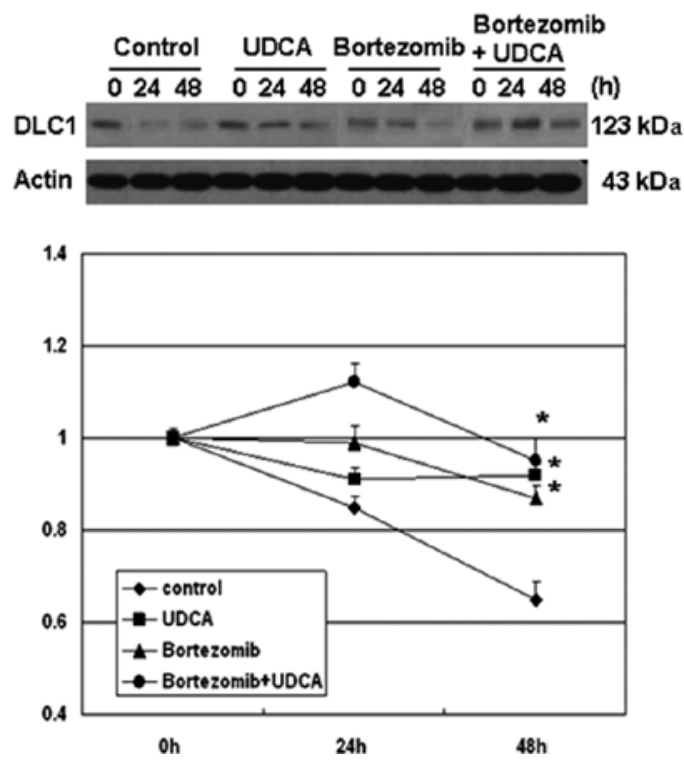

(B)

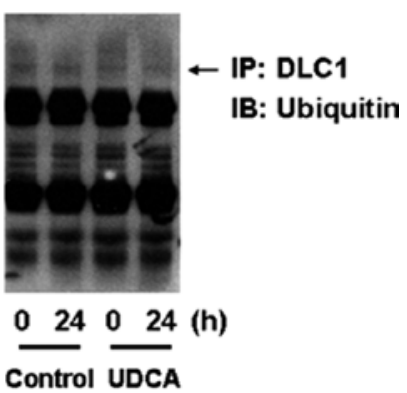

Figure 3. UDCA increases DLC1 protein stability. (A) SNU761 cells were treated with media (control), UDCA (200 $\mu \mathrm{M})$ or a proteasome inhibitor, bortezomib $(0.1 \mu \mathrm{M})$ in the presence of cycloheximide $(20 \mu \mathrm{M} / \mathrm{ml})$. At each time point, cells were lysed and immunoblot analysis was performed for DLC1 and $\beta$-actin. The ratios were calculated by densitometric scanning of the intensity of the DLC1 band relative to the $\beta$-actin band. Data representing the mean and SD from triplicate experiments are presented in the bar graph. "P $<0.05$, versus control. (B) SNU761 cells were treated with UDCA (200 $\mu$ M). Immunoprecipitation of DLC1 from cell lysates was performed followed by immunoblot analyses using either anti-ubiquitin or anti-DLC1 antibody.

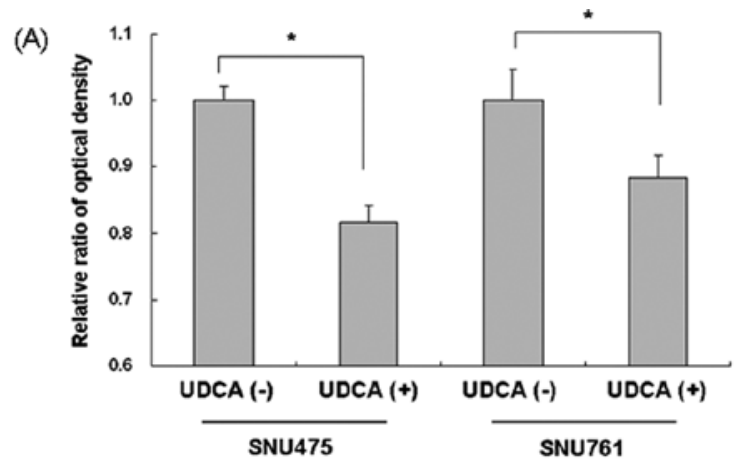

(B)

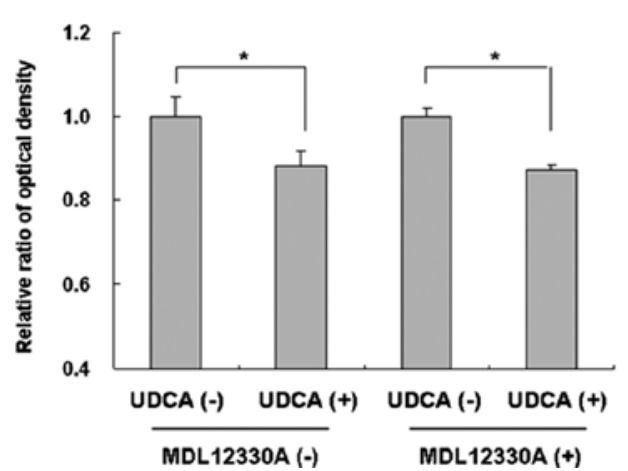

(C)

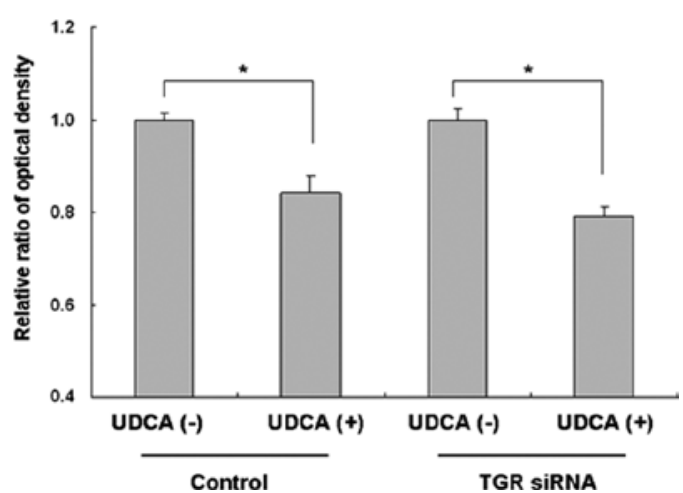

Figure 4. The effect of UDCA on HCC cellular proliferation. (A) SNU475 and SNU761 cells were cultured in the presence of UDCA (200 $\mu$ M). MTS (Promega) assays were performed at $24 \mathrm{~h}$, as instructed by the manufacturer. Data representing the mean and SD from triplicate experiments are presented in the bar graph. "P<0.05. (B) SNU761 cells were treated with an adenylate cyclase inhibitor, MDL12330A (10 $\mu \mathrm{M})$ and UDCA (200 $\mu \mathrm{M})$. MTS assays were performed at $24 \mathrm{~h}$. Data representing the mean and SD from triplicate experiments are presented in the bar graph. "P $<0.05$. (C) Twenty-four hours after transfection of either control or TGR5-siRNA, SNU761 cells were treated with UDCA (200 $\mu \mathrm{M})$. MTS assays were performed at $48 \mathrm{~h}$. Data representing the mean and SD from triplicate experiments are presented in the bar graph. ${ }^{*} \mathrm{P}<0.05$.

p42/44 MAPK activation was more enhanced while JNK was less activated in DLC1 specific siRNA-transfected cells than in control siRNA-transfected cells (Fig. 5B). These findings suggest that DLC1 is responsible for reducing p42/44 MAPK activation and enhancing JNK activation following UDCA treatment. 
(A)

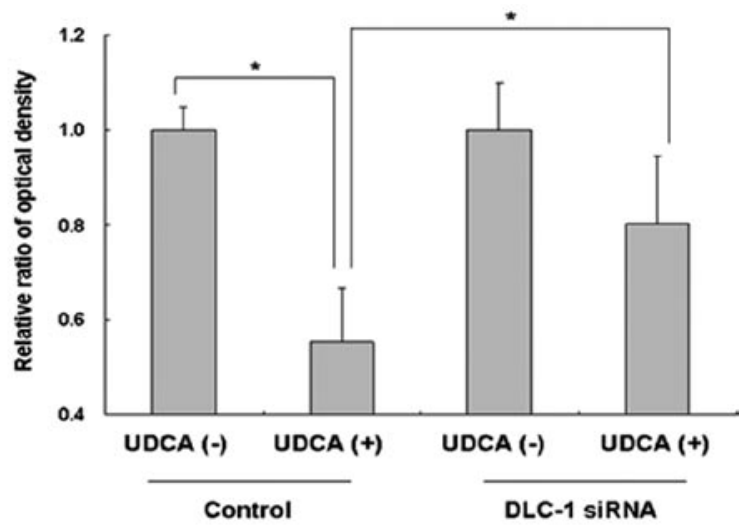

(B)

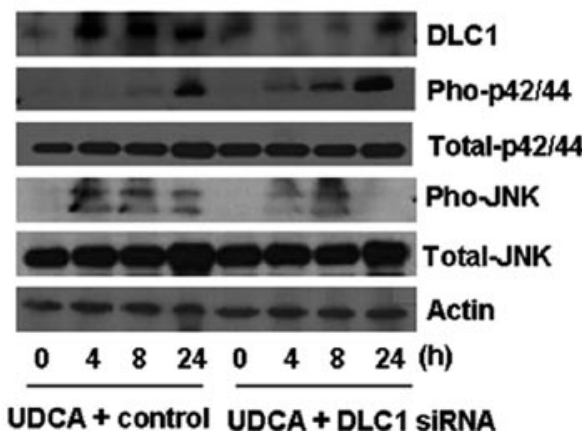

Figure 5. The effect of DLC1 on UDCA-induced growth suppression. (A) Twenty-four hours after transfection of either the control or DLC1 siRNA, SNU761 cells were treated with UDCA $(200 \mu \mathrm{M})$. MTS (Promega) assays were performed at $24 \mathrm{~h}$. Data representing the mean and SD from triplicate experiments are presented in the bar graph. ${ }^{*} \mathrm{P}<0.05$. (B) SNU761 cells were cultured in the presence of UDCA $(200 \mu \mathrm{M})$ for the indicated intervals after transfection of either the control or DLC1-specific siRNA. Equivalent amounts of protein were immunoblotted with anti-sera specific for DLC1 and the total and phosphorylated forms of p42/44 MAPK (Pho-p42/44) and JNK (Pho-JNK). Immunoblot using anti-actin antisera was performed as a control for protein loading.

To further determine whether p42/44 MAPK or JNK activation is responsible for DLC1-dependent growth suppression, we utilized both kinase signal inhibitors. Treatment of HCC cells with U0126 (p42/44 MAPK inhibitor) did not suppress the cellular growth, and SP600125 (JNK inhibitor) did not increase the growth of HCC cells (data not shown). These results indicate that UDCA-induced alteration of these kinase activities does not play a role in $\mathrm{HCC}$ cell growth suppression.

Involvement of RhoA activation in UDCA-induced growth suppression in HCC cells. Previous observations found that DLC1 growth suppression is mediated in a RhoGAPdependent and -independent manner (23). To assess the importance of RhoA activity in UDCA-induced growth suppression, we utilized a Rho inhibitor, Clostridium difficile toxin B-10463 (TcdB-10463), that inhibits RhoA/Rac1/Cdc42 (19). As shown in Fig. 6A, increased cellular proliferation after transfection of DLC1-siRNA in UDCA-treated HCC cells was reduced following Rho inhibitor treatment. Next, we examined whether UDCA leads to alteration of RhoA activity in HCC cells. As shown in Fig. 6B, RhoA activity was reduced following treatment with UDCA and the decreased RhoA activity induced by UDCA was reversed and thus increased following DLC1-siRNA transfection. These observations indicate that RhoA activity participates in DLC1-dependent growth suppression induced by UDCA in HCC cells.

\section{Discussion}

The principal finding of this study relates to the effect of UDCA on DLC1 protein expression and growth suppression in human HCC cells. The results demonstrate that a) UDCA inhibits DLC1 protein degradation in HCC cells resulting in increased expression of DLC1 and b) increased cellular DLC1 protein by UDCA suppresses HCC cellular growth. Theses observations provide new information regarding the anti-proliferative effect of UDCA in HCC cells.

We demonstrated that UDCA increases DLC1 protein levels in HCC cells and thereby suppresses cellular growth in a RhoA-dependent manner. Among the bile acids tested, UDCA was more effective than tauro-conjugates and chenodeoxycholic bile acid in increasing DLC1 protein levels (data not shown). DLC1 induction in our study was consistent with previous observations. For example, a histone deacetylase inhibitor induced DLC1 mRNA expression in gastric cell lines (11), and a dietary constituent, flavone, increased mRNA expression of DLC1 in human breast cancer cells and colon carcinoma cells (24). Our data showing the induction of DLC1 at the protein level by UDCA is a novel finding. Maintenance of cellular protein levels includes the interaction of transcriptional, translational and posttranslational regulation. As assessed by MSP, UDCA did not alter the methylation status of the DLC1 promoter. These results implicate an additional process other than promoter methylation, namely, inhibition of the turnover of DLC1 protein by UDCA. Our current data showed that UDCA inhibited DLC1 protein degradation in HCC cells. The mechanism of DLC1 induction by UDCA is in accordance with our previous finding that bile acids increased cellular Mcl-1 protein levels by inhibiting Mcl-1 degradation (2).

In the current study, UDCA did not modulate the DLC1polyubiquitin conjugates, whereas the selective proteasome inhibitor, bortezomib, increased cellular DLC1 protein levels. These findings suggest that UDCA induces DLC1 protein by inhibiting proteasomal DLC1 degradation in an ubiquitin-independent manner. Many proteins become polyubiquitinated as a signal for selective proteasomal degradation (21). This ubiquitin conjugation to proteins is a reversible process, and this process is achieved by deubiquitinating enzymes (DUBs) consisting of ubiquitin $\mathrm{COOH}$-terminal hydrolases (UCHs) and ubiquitin-specific proteases (USPs or UBPs). DUBs can direct the turnover of specific proteins by removing ubiquitin or polyubiquitin chains from the substrate to avoid their degradation by the proteasome (25). USP activity on the ubiquitin chains is suggested to function as a timer; if the chain becomes too short before the substrate is fully engaged, the protein escapes degradation until it is again ubiquitinated for another round (26). UDCA may participate in intramolecular interactions between DLC1 and USPs. RhoBTB proteins, which are members of the Rho family of small GTPases, were reported to interact with Cullins, which function as scaffolding proteins that bring together the ubiquitin conjugating enzyme and substrate recognition 
(B)

(A)

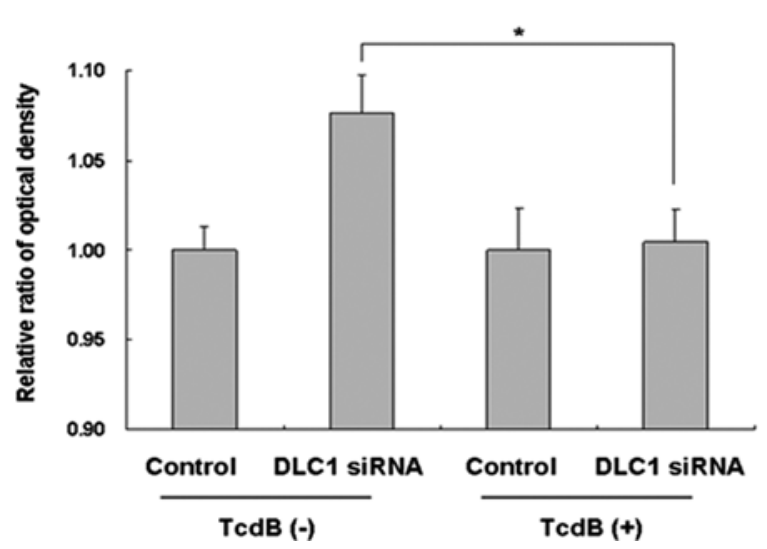

RhoA
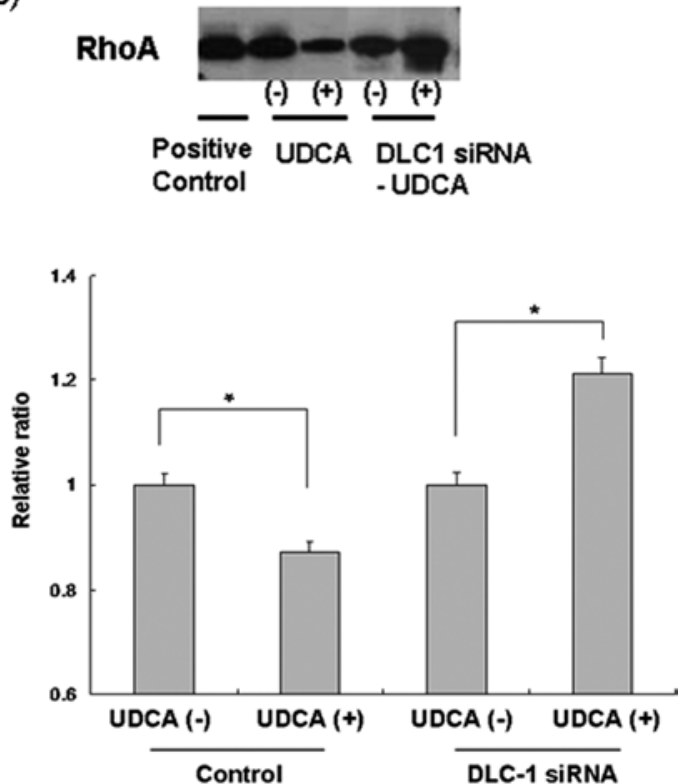

Figure 6. The effect of a Rho-inhibitor on UDCA-induced growth suppression. (A) Twenty-four hours after transfection of either the control or DLC1-siRNA, SNU761 cells were treated with a Rho-inhibitor, TcdB10642 $(50 \mu \mathrm{M})$ and UDCA $(200 \mu \mathrm{M})$. MTS (Promega) assays were performed at $24 \mathrm{~h}$, as instructed by the manufacturer. Data representing the mean and SD from triplicate experiments are presented in the bar graph. *P<0.05. (B) SNU761 cells were treated with UDCA $(200 \mu \mathrm{M})$. RhoA levels were assessed by incubating lysates with GST-Rhotekin-RBD that was precoupled to glutathione-sepharose beads Precipitated and total lysates were analyzed by Western blotting with anti-RhoA antibody. Twenty-four hours after transfection of either the control or DLC1-siRNA, SNU761 cells were treated with UDCA $(200 \mu \mathrm{M})$. RhoA levels were assessed as described above. The ratios were calculated by densitometric scanning of the intensity of the UDCA-treated RhoA band relative to the untreated band. Data representing the mean and SD from triplicate experiments are presented in the bar graph. ${ }^{*} \mathrm{P}<0.05$.

component for ubiquitin mediated degradation by the $26 \mathrm{~S}$ proteasome (27). However, more studies are required to determine the mechanism by which UDCA inhibits DLC1 proteasomal degradation.

UDCA is known to protect hepatocytes from bile acidsinduced apoptosis via activation of survival pathways (5). It also regulates genetic transcription related to apoptosis, the cell cycle, and cellular proliferation (28). For example, UDCA blocked carcinogen-induced alterations in cyclin D1 and E-cadherin protein in a colon cancer model (29). However, the effect of UDCA on human HCC cells has not yet been elucidated yet. It has recently been reported that UDCA selectively inhibited cellular proliferation and induced apoptosis in human hepatoma cell lines by blocking the cell cycle and regulating Bax/bcl-2 gene expression (30). In another experiment, the addition of UDCA into human Huh-7 and rat Fao HCC cells induced apoptosis in a dose-dependent manner (31). In the current study, UDCA significantly suppressed cellular proliferation in both HCC cell lines and the growth suppression was significantly reversed following inhibition of DLC1. Therefore, it is likely that UDCA reduces cellular proliferation via a DLC1-dependent pathway.

Although UDCA-induced kinase activation, which included more enhanced p42/44 MAPK activation and less JNK activation, was altered by inhibition of DLC1, inhibition of p42/44 MAPK or JNK did not alter the growth suppression by UDCA in our study. It has previously been demonstrated that DLC1 decreases RhoA activity, which may lead to the reduced ability to migrate (23). Inhibition of Rho significantly reduced the increase in cellular proliferation by
DLC1-siRNA transfection in UDCA-treated cells. Moreover, RhoA activity was reduced following treatment with UDCA, and inhibition of DLC1 by siRNA transfection increased RhoA activity. Taken together, RhoA activity may participate in UDCA-induced DLC1-dependent growth suppression. On the other hand, UDCA-induced growth suppression was not affected by blocking cell surface TGR 5 activation. Thus, it is more likely that other signals, which are activated by intracellularly transported UDCA, might be mediating this growth suppressing effect.

In summary, the findings of the present study demonstrate that UDCA induces DLC1 protein expression by inhibiting proteasomal DLC1 degradation in a ubiquitin-independent manner, and DLC1 induction participates in UDCA-induced suppression of HCC cellular growth. Therefore, UDCA may have therapeutic implication as an anti-proliferative agent in HCCs.

\section{Acknowledgements}

This study was funded by the Korea Health 21 R\&D Project (No. 0412-CR01-0704-0001).

\section{References}

1. Debruyne PR, Bruyneel EA, Karaguni IM, et al: Bile acids stimulate invasion and haptotaxis in human colorectal cancer cells through activation of multiple oncogenic signaling pathways. Oncogene 21: 6740-6750, 2002.

2. Yoon JH, Werneburg NW, Higuchi $\mathrm{H}$, et al: Bile acids inhibit Mcl-1 protein turnover via an epidermal growth factor receptor/ Raf-1-dependent mechanism. Cancer Res 62: 6500-6505, 2002. 
3. Yoon JH, Higuchi H, Werneburg NW, Kaufmann SH and Gores GJ: Bile acids induce cyclooxygenase-2 expression via the epidermal growth factor receptor in a human cholangiocarcinoma cell line. Gastroenterology 122: 985-993, 2002.

4. Martinez-Diez MC, Serrano MA, Monte MJ and Marin JJ: Comparison of the effects of bile acids on cell viability and DNA synthesis by rat hepatocytes in primary culture. Biochim Biophys Acta 1500: 153-160, 2000.

5. Schoemaker MH, Conde de la Rosa L, Buist-Homan M, et al: Tauroursodeoxycholic acid protects rat hepatocytes from bile acid-induced apoptosis via activation of survival pathways. Hepatology 39: 1563-1573, 2004.

6. Khare S, Cerda S, Wali RK, et al: Ursodeoxycholic acid inhibits Ras mutations, wild-type Ras activation, and cyclooxygenase-2 expression in colon cancer. Cancer Res 63: 3517-3523, 2003.

7. Yuan BZ, Miller MJ, Keck CL, Zimonjic DB, Thorgeirsson SS and Popescu NC: Cloning, characterization, and chromosomal localization of a gene frequently deleted in human liver cancer (DLC-1) homologous to rat RhoGAP. Cancer Res 58: 2196-2199, 1998.

8. Morii N, Kumagai N, Nur EKMS, Narumiya S and Maruta H: rho GAP of $28 \mathrm{kDa}$ (GAP2), but not of $190 \mathrm{kDa}$ (p190), requires Asp65 and Asp67 of rho GTPase for its activation. J Biol Chem 268: 27160-27163, 1993.

9. Ullmannova V and Popescu NC: Inhibition of cell proliferation, induction of apoptosis, reactivation of DLC1, and modulation of other gene expression by dietary flavone in breast cancer cell lines. Cancer Detect Prev 31: 110-118, 2007.

10. Ng IO, Liang ZD, Cao L and Lee TK: DLC-1 is deleted in primary hepatocellular carcinoma and exerts inhibitory effects on the proliferation of hepatoma cell lines with deleted DLC-1. Cancer Res 60: 6581-6584, 2000.

11. Kim TY, Jong HS, Song SH, et al: Transcriptional silencing of the DLC-1 tumor suppressor gene by epigenetic mechanism in gastric cancer cells. Oncogene 22: 3943-3951, 2003.

12. Yuan BZ, Durkin ME and Popescu NC: Promoter hypermethylation of DLC-1, a candidate tumor suppressor gene, in several common human cancers. Cancer Genet Cytogenet 140: 113-117, 2003.

13. Kim TY, Lee JW, Kim HP, Jong HS, Jung M and Bang YJ: DLC-1, a GTPase-activating protein for Rho, is associated with cell proliferation, morphology, and migration in human hepatocellular carcinoma. Biochem Biophys Res Commun 355 72-77, 2007.

14. Wong CM, Yam JW, Ching YP, et al: Rho GTPase-activating protein deleted in liver cancer suppresses cell proliferation and invasion in hepatocellular carcinoma. Cancer Res 65: 8861-8868, 2005.

15. Yoon JH, Lee HV, Lee JS, Park JB and Kim CY: Development of a non-transformed human liver cell line with differentiatedhepatocyte and urea-synthetic functions: applicable for bioartificial liver. Int J Artif Organs 22: 769-777, 1999.

16. Faubion WA, Guicciardi ME, Miyoshi H, et al: Toxic bile salts induce rodent hepatocyte apoptosis via direct activation of Fas. J Clin Invest 103: 137-145, 1999.
17. Zhou X, Thorgeirsson SS and Popescu NC: Restoration of DLC-1 gene expression induces apoptosis and inhibits both cell growth and tumorigenicity in human hepatocellular carcinoma cells. Oncogene 23: 1308-1313, 2004.

18. Wong CM, Lee JM, Ching YP, Jin DY and Ng IO: Genetic and epigenetic alterations of DLC-1 gene in hepatocellular carcinoma. Cancer Res 63: 7646-7651, 2003.

19. Just I, Selzer J, Wilm M, von Eichel-Streiber C, Mann M and Aktories K: Glucosylation of Rho proteins by Clostridium difficile toxin B. Nature 375: 500-503, 1995.

20. Reuther GW, Lambert QT, Booden MA, et al: Leukemiaassociated Rho guanine nucleotide exchange factor, a Dbl family protein found mutated in leukemia, causes transformation by activation of RhoA. J Biol Chem 276: 27145-27151, 2001.

21. Mayer RJ: The meteoric rise of regulated intracellular proteolysis. Nat Rev Mol Cell Biol 1: 145-148, 2000.

22. Kawamata Y, Fujii R, Hosoya M, et al: A G protein-coupled receptor responsive to bile acids. J Biol Chem 278: 9435-9440, 2003.

23. Healy KD, Hodgson L, Kim TY, et al: DLC-1 suppresses non-small cell lung cancer growth and invasion by RhoGAPdependent and independent mechanisms. Mol Carcinog 47: 326-337, 2008

24. Herzog A, Kindermann B, Doring F, Daniel H and Wenzel U: Pleiotropic molecular effects of the pro-apoptotic dietary constituent flavone in human colon cancer cells identified by protein and mRNA expression profiling. Proteomics 4: 2455-2464, 2004.

25. Jung T, Catalgol B and Grune T: The proteasomal system. Mol Aspects Med 30: 191-296, 2009.

26. Kraut DA, Prakash S and Matouschek A: To degrade or release: ubiquitin-chain remodeling. Trends Cell Biol 17: 419-421, 2007.

27. Berthold J, Schenkova K, Ramos S, et al: Characterization of RhoBTB-dependent Cul3 ubiquitin ligase complexes - evidence for an autoregulatory mechanism. Exp Cell Res 314: 3453-3465, 2008.

28. Castro RE, Sola S, Ma X, et al: A distinct microarray gene expression profile in primary rat hepatocytes incubated with ursodeoxycholic acid. J Hepatol 42: 897-906, 2005.

29. Wali RK, Khare S, Tretiakova M, et al: Ursodeoxycholic acid and $F(6)-D(3)$ inhibit aberrant crypt proliferation in the rat azoxymethane model of colon cancer: roles of cyclin D1 and E-cadherin. Cancer Epidemiol Biomarkers Prev 11: 1653-1662, 2002.

30. Liu H, Qin CY, Han GQ, Xu HW, Meng M and Yang Z: Mechanism of apoptotic effects induced selectively by ursodeoxycholic acid on human hepatoma cell lines. World J Gastroenterol 13: 1652-1658, 2007.

31. Oyama K, Shiota G, Ito H, Murawaki Y and Kawasaki H: Reduction of hepatocarcinogenesis by ursodeoxycholic acid in rats. Carcinogenesis 23: 885-892, 2002. 